\title{
Fatores associados ao conhecimento e atitudes de acadêmicos de enfermagem sobre comportamento sexual e Infecções Sexualmente Transmissíveis em pessoas idosas
}

\author{
Factors associated with the knowledge and attitudes of nursing students about sexual behavior and \\ Sexually Transmitted Infections in the elderly
}

Factores asociados a los conocimientos y actitudes de los estudiantes de enfermería sobre el comportamiento sexual y las Infecciones de Transmisión Sexual en el anciano

Recebido: 24/01/2022 | Revisado: 01/02/2022 | Aceito: 10/02/2022 | Publicado: 15/02/2022

Ana Carolina Macri Gaspar Vendramini
ORCID: https://orcid.org/0000-0001-5712-7115
Universidade do Estado de Mato Grosso, Brasil
E-mail: anacarolinamacri@ @otmail.com.br
Priscila Aguiar Mendes
ORCID: https://orcid.org/0000-0002-0252-091X
Universidade do Estado de Mato Grosso, Brasil
E-mail: prih.mendes@ gmail.com
Annelita Almeida Oliveira Reiners
ORCID: https://orcid.org/0000-0002-5699-8215
Universidade Federal do Mato Grosso, Brasil
E-mail:annereiners.ar@ gmail.com
Amanda Cristina de Souza Andrade
ORCID: https://orcid.org/0000-0002-3366-4423
Universidade Federal do Mato Grosso, Brasil
E-mail:csouza.amanda @ gmail.com
Rosemeiry Capriata De Souza Azevedo
ORCID: https://orcid.org/0000-0001-7986-5768
Universidade Federal do Mato Grosso, Brasil
E-mail:rosemeirycapriataazevedo@ gmail.com

\begin{abstract}
Resumo
Analisar os fatores sociodemográficos e de formação acadêmica associados ao conhecimento e às atitudes de acadêmicos de enfermagem sobre o comportamento sexual e as Infecções Sexualmente Transmissíveis em pessoas idosas. Estudo transversal, realizado com 88 acadêmicos de enfermagem matriculados do quinto ao décimo semestre de uma universidade pública. A coleta de dados ocorreu em 2020, utilizando questionário online autoaplicável sobre características sociodemográficas, formação acadêmica, conhecimentos e atitudes sobre o comportamento sexual e IST em pessoas idosas. Na análise descritiva, calcularam-se frequências absolutas e relativas para as variáveis categóricas. Para o conhecimento e a atitude, calcularam-se média, mediana, distância interquartil e desvio-padrão conforme as variáveis sociodemográficas e de formação acadêmica. Realizou-se regressão linear e múltipla para avaliar as variáveis associadas ao conhecimento e às atitudes, adotando nível de significância de 5\%. As variáveis associadas ao conhecimento dos acadêmicos foram: faixa etária de 27 a 39 anos (beta=1,45; IC95\%:0,18-2,07); contato com idosos (beta=1,06; IC95\%:0,48-2,07); atividade complementar IST sobre pessoas idosas (beta=2,43; IC95\%:1,01-3,84); sétimo (beta=2,22; IC95\%:1,08-3,37) e nono (beta=1,67; IC95\%:0,52-2,82) semestres em curso. Quanto às atitudes, participar em atividades complementares se manteve associada no modelo múltiplo (beta=10,24; IC95\%:1,17-19,31). Acadêmicos de enfermagem com faixa etária de 27 anos ou mais, que têm contato próximo com idosos, que cursam entre o sétimo e décimo semestres e que já participaram de atividades complementares têm maior nível de conhecimento sobre comportamento sexual e as IST em pessoas idosas. Aqueles que participaram de atividades complementares têm atitudes mais positivas.
\end{abstract}

Palavras-chave: Estudantes de enfermagem; Idoso; Doenças sexualmente transmissíveis; Sexualidade.

\begin{abstract}
To analyze the sociodemographic and academic background factors associated with the knowledge and attitudes of nursing students about sexual behavior and Sexually Transmitted Infections in the elderly. Cross-sectional study, carried out with 88 nursing students enrolled from the fifth to the tenth semester of a public university. Data collection took place in 2020, using a self-administered online questionnaire on sociodemographic characteristics, academic background, knowledge and attitudes about sexual behavior and STIs in older people. In the descriptive analysis, absolute and relative frequencies were calculated for the categorical variables. For knowledge and attitude, mean, median, interquartile distance and standard deviation were calculated according to sociodemographic and academic
\end{abstract}


background variables. Linear and multiple regression were performed to assess variables associated with knowledge and attitudes, adopting a significance level of 5\%. The variables associated with the students' knowledge were: age group from 27 to 39 years (beta=1.45; 95\%CI:0.18-2.07); contact with the elderly (beta=1.06; 95\%CI:0.48-2.07); STI complementary activity on elderly people (beta=2.43; 95\%CI:1.01-3.84); seventh (beta=2.22; 95\%CI:1.08-3.37) and ninth (beta $=1.67 ; 95 \% \mathrm{CI}: 0.52-2.82$ ) ongoing semesters. As for attitudes, participating in complementary activities remained associated in the multiple model (beta=10.24; 95\%CI:1.17-19.31). Nursing students aged 27 years or older, who have close contact with the elderly, who attend between the seventh and tenth semesters and who have already participated in complementary activities have a higher level of knowledge about sexual behavior and STIs in elderly people. Those who participated in complementary activities have more positive attitudes

Keywords: Nursing students; Aged; Sexually transmitted diseases; Sexuality.

\section{Resumen}

Analizar los factores sociodemográficos y de formación académica asociados a los conocimientos y actitudes de los estudiantes de enfermería sobre el comportamiento sexual y las Infecciones de Transmisión Sexual en los ancianos. Estudio de corte transversal, realizado con 88 estudiantes de enfermería matriculados del quinto al décimo semestre de una universidad pública. La recolección de datos se llevó a cabo en 2020, mediante un cuestionario en línea autoadministrado sobre características sociodemográficas, formación académica, conocimientos y actitudes sobre el comportamiento sexual y las ITS en personas mayores. En el análisis descriptivo se calcularon frecuencias absolutas y relativas para las variables categóricas. Para conocimiento y actitud se calculó media, mediana, distancia intercuartil y desviación estándar según variables sociodemográficas y de formación académica. Se realizaron regresiones lineales y múltiples para evaluar variables asociadas a conocimientos y actitudes, adoptando un nivel de significación del 5\%. Las variables asociadas al conocimiento de los estudiantes fueron: grupo etario de 27 a 39 años (beta=1,45; IC95\%:0,18-2,07); contacto con ancianos (beta=1,06; IC95\%:0,48-2,07); Actividad complementaria de ITS en ancianos (beta=2,43; IC95\%:1,01-3,84); séptimo (beta=2,22; IC95\%:1,08-3,37) y noveno (beta=1,67;IC95\%:0,522,82) semestres en curso. En cuanto a las actitudes, la participación en actividades complementarias permaneció asociada en el modelo múltiple (beta=10,24; IC95\%:1,17-19,31). Los estudiantes de Enfermería con edad igual o superior a 27 años, que tienen contacto estrecho con el adulto mayor, que cursan entre el séptimo y décimo semestre y que ya han participado en actividades complementarias, tienen mayor nivel de conocimiento sobre el comportamiento sexual y las ITS en el adulto mayor. Quienes participaron en actividades complementarias tienen actitudes más positivas.

Palabras clave: Estudiantes de enfermería; Anciano; Enfermedades de transmisión sexual; Sexualidad.

\section{Introdução}

Pesquisas mostram um aumento exponencial de Infecções Sexualmente Transmissíveis (IST) na população idosa em diversos países (Brasil, 2020; Linley et al., 2018; European Centre for Disease Prevention And Control [ECDPC], 2020; Brasil, 2021; Joint United Nations Programme on Hiv/Aids [UNAIDS], 2016). Globalmente, em 2016, 17\% dos adultos que viviam com HIV tinham 50 anos ou mais (UNAIDS, 2016). A proporção de casos de Sífilis em pessoas com 45 anos ou mais têm aumentado nos últimos anos na Europa, representando 30\% dos casos notificados em 2018 (ECDPC, 2020). No Brasil, em 2021, 14,4\% dos casos notificados de Sífilis adquirida foram em pessoas com 50 anos ou mais (Brasil, 2021).

Alguns fatores têm contribuído para o aumento dos casos de IST nas pessoas mais velhas, como a prática sexual desprotegida (Andrade et al.,2017; Madruga et al., 2018; Moura, Pessôa et al., 2017) relacionamentos com parceiros casuais (Yang et al., 2018) e baixa percepção de risco (Madruga et al., 2018; Moura et al.,2017). Nesse contexto, é fundamental que profissionais de saúde incluam a saúde sexual no atendimento realizado às pessoas idosas. Entretanto, a literatura tem evidenciado que o cuidado sexual não é abordado pelos profissionais nos serviços de saúde (Agochukwu-Mmonu et al., 2021; Cunha et al., 2015; Dos Reis et al., 2020).

Estudos mostram que um dos motivos que dificultam essa abordagem são barreiras, como tabus e estigmas (Cunha et al., 2015; Dos Reis et al., 2020; Mahmud et al., 2021; Evangelista et al., 2019; Gaspar et al., 2020; Venturini et al., 2018) e crenças de que as pessoas mais velhas não possuem vida sexual ativa (Moura et al., 2017, Mahmud et al., 2021; Cunha et al., 2015). Além disso, há evidências de que é insuficiente o conhecimento desses profissionais sobre o comportamento sexual de pessoas idosas e suas atitudes em relação à expressão do seu desejo sexual, geralmente, são negativas (Mahmud et al., 2021; Evangelista et al., 2019). 
Assim, estudos apontam para a deficiência na formação de acadêmicos da área da saúde com respeito ao Comportamento Sexual dos Idosos (CSI) e também às IST (Costa et al., 2017; Moreira et al., 2018). Nos currículos, esse conteúdo é limitado (Cappiello et al., 2017) e não é considerado prioridade (Moreira et al., 2018). Pesquisas realizadas com acadêmicos da área da saúde são, em sua maioria, internacionais e têm demonstrado que eles possuem pouco conhecimento sobre comportamento sexual e IST em pessoas idosas e que suas atitudes são negativas (Chu et al., 2020; Gewirtz-Meydan et al., 2018; Wilschut et al., 2021).

Investigações internacionais e nacionais realizadas com acadêmicos de enfermagem sobre saúde sexual de pessoas idosas são escassas. Nesse sentido, na literatura não há consenso em relação aos fatores associados ao conhecimento e às atitudes de acadêmicos de enfermagem em relação à sexualidade na velhice. Do que se sabe, a idade de acadêmicos, o contato prévio com pessoas idosas (Chu et al., 2020; Gewirtz-Meydan et al., 2018) e o período do curso Wilschut et al., 2021) estão associados ao conhecimento dos acadêmicos. Em relação às atitudes, idade, contato prévio com pessoas idosas 21 e período do curso (Chu et al., 2020) também foram associados. Dentre os estudos encontrados com acadêmicos de enfermagem sobre sexualidade de pessoas idosas, nenhum deles incluiu conhecimento e atitudes relacionadas às IST(Chu et al., 2020; GewirtzMeydan et al., 2018; Wilschut et al., 2021).

Assim, o objetivo foi analisar os fatores associados ao conhecimento e à atitude de acadêmicos de enfermagem sobre o comportamento sexual e as IST em pessoas idosas.

\section{Metodologia}

Estudo transversal analítico, realizado em um campus de uma universidade pública do estado de Mato Grosso. A população considerada para este estudo foi de 118 acadêmicos matriculados do quinto ao décimo semestre do curso de Enfermagem de Tangará da Serra (MT). Esse critério foi estabelecido em virtude de que a partir do quinto semestre os alunos já cursaram disciplinas que abordam a saúde do adulto e do idoso. Foi utilizada amostragem por conveniência. Incluíram-se alunos de ambos os sexos, com idade superior a 18 anos. Todos os alunos foram convidados a participar da pesquisa, porém, dentre aqueles que atenderam a esses critérios, 30 não aceitaram participar da pesquisa. Assim, a amostra final foi de 88 participantes.

A coleta de dados foi realizada em novembro de 2020. Encaminhou-se aos acadêmicos um e-mail contendo convite para participar da pesquisa, o Termo de Consentimento Livre e Esclarecido (TCLE) assinado pela pesquisadora e o questionário por meio de um link de acesso na plataforma Google Forms ${ }^{\circledR}$. A fim de minimizar o risco do email encaminhado ser endereçado a pastas de lixo eletrônico, a pesquisadora utilizou email institucional dos alunos como endereço para envio. O questionário permaneceu aberto para respostas no período de 30 dias e semanalmente a pesquisadora encaminhava novamente o convite para incentivar a participação dos alunos. Aqueles que não responderam neste período foram considerados como recusa, e representaram $25 \%$ do total da população.

O questionário foi online autoaplicável composto por uma página inicial com o TCLE e demais páginas com perguntas fechadas sobre as características sociodemográficas e a formação acadêmica. Outro instrumento encaminhado foi o de conhecimentos e atitudes sobre o comportamento sexual e as IST em pessoas idosas, criado pela pesquisadora e submetido a um comitê de oito especialistas para validação de conteúdo. O Índice de Validade de Conteúdo (IVC) foi de $96 \%$.

A primeira parte do instrumento sobre o conhecimento é formada por 13 questões com opções de respostas sim, não e não sei, cada uma valendo um ponto. As questões avaliam o conhecimento que os acadêmicos têm sobre o comportamento sexual das pessoas idosas (por exemplo: pessoas idosas têm vida sexual ativa, o processo de envelhecimento promove mudanças fisiológicas que influenciam no desempenho sexual das pessoas idosas; o desejo sexual permanece na velhice; a frequência da atividade sexual reduz com a velhice; atividade sexual de idosos inclui beijos/abraços/carícias, não somente a 
relação sexual; eles utilizam medicamentos para melhorar o desempenho sexual, eles utilizam brinquedos ou filmes eróticos; eles possuem relacionamentos casuais, mulheres idosas avaliam sua satisfação sexual de maneira mais positiva que os homens idosos) como também, o conhecimento sobre as IST nessa população (por exemplo: idosos usam preservativo nas relações sexuais, há muitos casos de IST nessa população; o diagnóstico de IST nessa população é tardio; existe políticas de prevenção de IST para especifica para pessoas idosas no país). Para cada resposta correta, soma-se um ponto. Ao final, o instrumento permite avaliar um escore que pode variar entre 0 a 13 pontos. Quanto maior o escore, maior o conhecimento que os acadêmicos têm sobre o comportamento sexual e IST de pessoas idosas.

A segunda parte do instrumento é sobre às atitudes, sendo constituído por 26 questões que abrangem os três componentes da atitude, cognitivo, afetivo e comportamental (Altmann, 2008). As atitudes foram denominadas como uma reação psicológica expressa por avaliações positivas ou negativas, mensuradas por meio das respostas elencadas em cada afirmativa do questionário, que foram consideradas como um estímulo à atitude, uma vez que, apresentam situações acerca do comportamento sexual e IST em pessoas idosas (por exemplo, afirmativas sobre o componente cognitivo: "acredito que a maioria das pessoas idosas deixa de se interessar por sexo"; "masturbação é apenas para jovens"; "é vergonhoso um idoso demonstrar interesse por sexo"; "não acredito que pessoas idosas também têm IST". Componentes afetivos: "sinto-me constrangido ao conversar com pessoas idosas sobre seu comportamento sexual ou sobre IST"; confio na minha capacidade técnica para falar sobre IST e sua prevenção com pessoas idosas"; "tenho vergonha de ensinar um idoso a utilizar o preservativo". Em relação aos componentes comportamentais: "reservaria um tempo da consulta de enfermagem para falar sobre comportamento sexual com o idoso"; "desenvolveria ações de educação em saúde sobre prevenção de IST com pessoas idosas"). As opções de respostas são do tipo Likert, variando entre concordo totalmente a discordo totalmente, e pontuações de 1 a 5 pontos. O escore final varia entre 1 e 130 pontos. Quanto maior o escore, mais positivas são as atitudes dos acadêmicos em relação ao comportamento sexual e IST em pessoas idosas.

As variáveis dependentes do estudo são o conhecimento e as atitudes dos acadêmicos de enfermagem sobre o comportamento sexual e as IST de pessoas idosas analisadas pelo escore. Já as variáveis independentes são relacionadas às características sociodemográficas (sexo, faixa etária, estado civil, religião, contato próximo com idosos) e de formação acadêmica (semestre em curso, conteúdo sobre CSI, conteúdo sobre IST em idosos, atividade complementar sobre CS e IST, pesquisa com idosos, extensão com idosos, prática assistencial com idosos).

Para organização dos dados, foi criado um banco no software Excel® com os dados exportados a partir da planilha gerada pela plataforma google forms ${ }^{\circledR}$. Em seguida, o banco foi exportado para o programa estatístico Stata ${ }^{\circledR}$ versão 16 para realização da análise dos dados. $\mathrm{Na}$ análise descritiva, calcularam-se frequências absolutas e relativas para as variáveis categóricas. Para o conhecimento e a atitude, calcularam-se média, mediana, distância interquartil e desvio-padrão conforme as variáveis sociodemográficas e de formação acadêmica. Para avaliar a associação bivariada e multivariada entre o conhecimento e atitude e as variáveis independentes, utilizou-se a regressão linear com a técnica stepwise forward. As variáveis que apresentaram $\mathrm{p}<0,20$ na análise bivariada foram incluídas no modelo múltiplo e se mantiveram no modelo final aquelas com $\mathrm{p}<0,05$. As suposições de normalidade, linearidade e homocedasticidade do modelo foram verificadas a partir da análise de resíduos. Adotou-se o nível de significância de $5 \%$.

A pesquisa atendeu aos critérios estabelecidos pela resolução que orienta pesquisa com seres humanos. O projeto foi aprovado pelo Comitê de Ética em Pesquisa (CEP) com parecer número 3.924.902/2020, CAAE 29371420.0.0000.8124 e seguiu os preceitos éticos estabelecidos na resolução do Conselho Nacional de Pesquisa (CNS) com Seres Humanos. 


\section{Resultados}

Participaram do estudo 88 acadêmicos de enfermagem. Destes 89,8\% são do sexo feminino, 27,3\% têm idade entre 21 e 22 anos (média 25 anos, DP: 3,98), 67,1\% são solteiros e 38,6\% católicos ou protestantes. A maioria dos acadêmicos (75,0\%) referiu ter contato próximo com pessoas com 60 anos ou mais, principalmente com os avós.

Quanto à formação acadêmica, 28,4\% dos acadêmicos de enfermagem cursam o quinto semestre. A maioria deles referiu não ter tido o conteúdo sobre o CS ou as IST em pessoas idosas (62,5\% e 69,3\%, respectivamente) na graduação, não participou de atividades complementares, como palestras sobre CS $(89,9 \%)$ ou IST em pessoas idosas $(88,6 \%)$ e pesquisas $(85,2 \%)$ ou extensão $(73,9 \%)$ que envolvessem essa população; $64,8 \%$ realizaram prática assistencial com pessoas idosas sem abordar esse conteúdo.

Os resultados da análise bivariada mostram que o conhecimento foi maior entre os alunos de 27 a 39 anos $(p<0,001)$, do nono e décimo anos $(p<0,001)$, que receberam conteúdo sobre CSI $(p=0,032)$ e ISTI $(p=0,016)$, que realizaram atividades complementares CSI $(p=0,003)$ e STI $(p<0,001)$ e tiveram prática assistencial $(p<0,046)$ (Tabela 1$)$.

Tabela 1. Comparação do escore de conhecimento dos acadêmicos conforme variáveis sociodemográficas e de formação $(\mathrm{n}=88)$. Tangará da Serra, MT, 2020.

\begin{tabular}{|c|c|c|c|c|c|c|}
\hline Variáveis & $\mathbf{n}$ & Média & $\begin{array}{l}\text { Desvio- } \\
\text { Padrão }\end{array}$ & Mediana & $\begin{array}{c}\text { Distância } \\
\text { interquartil }\end{array}$ & $\mathbf{p}$ \\
\hline $\begin{array}{l}\text { Sexo } \\
\text { Feminino } \\
\text { Masculino } \\
\end{array}$ & $\begin{array}{r}79 \\
9 \\
\end{array}$ & $\begin{array}{l}8,44 \\
8,22 \\
\end{array}$ & $\begin{array}{l} \pm 2,52 \\
\pm 2,33 \\
\end{array}$ & $\begin{array}{l}9,0 \\
9,0 \\
\end{array}$ & $\begin{array}{l}4,0 \\
4,0 \\
\end{array}$ & 0,803 \\
\hline $\begin{array}{l}\text { Faixa etária } \\
20 \text { a } 22 \text { anos } \\
23 \text { a } 26 \text { anos } \\
27 \text { a } 39 \text { anos }\end{array}$ & $\begin{array}{l}36 \\
34 \\
18 \\
\end{array}$ & $\begin{array}{r}7,52 \\
8,38 \\
10,27 \\
\end{array}$ & $\begin{array}{l} \pm 2,65 \\
\pm 2,13 \\
\pm 1,77 \\
\end{array}$ & $\begin{array}{r}8,0 \\
8,5 \\
10,5 \\
\end{array}$ & $\begin{array}{l}4,0 \\
4,0 \\
3,0 \\
\end{array}$ & $<0,001$ \\
\hline $\begin{array}{l}\text { Estado civil } \\
\text { Sem companheiro } \\
\text { Com companheiro }\end{array}$ & $\begin{array}{l}59 \\
29 \\
\end{array}$ & $\begin{array}{l}8,15 \\
8,96 \\
\end{array}$ & $\begin{array}{c} \pm 2,48 \\
\pm \\
\end{array}$ & $\begin{array}{l}8,0 \\
9,0 \\
\end{array}$ & $\begin{array}{l}4,0 \\
3,0 \\
\end{array}$ & 0,151 \\
\hline $\begin{array}{l}\text { Religião } \\
\text { Católico } \\
\text { Protestante } \\
\text { Outra } \\
\text { Sem religião } \\
\end{array}$ & $\begin{array}{r}34 \\
34 \\
5 \\
15 \\
\end{array}$ & $\begin{array}{l}8,73 \\
8,70 \\
8,00 \\
7,20\end{array}$ & $\begin{array}{l} \pm 2,23 \\
\pm 2,54 \\
\pm 3,16 \\
\pm 2,59 \\
\end{array}$ & $\begin{array}{l}9,5 \\
9,0 \\
7,0 \\
7,0 \\
\end{array}$ & $\begin{array}{l}3,0 \\
4,0 \\
3,0 \\
4,0 \\
\end{array}$ & 0,194 \\
\hline $\begin{array}{l}\text { Contato próximo com pessoas idosas } \\
\text { Sim } \\
\text { Não }\end{array}$ & $\begin{array}{l}64 \\
24 \\
\end{array}$ & $\begin{array}{l}8,67 \\
7,75 \\
\end{array}$ & $\begin{array}{l} \pm 2,48 \\
\pm 2,45\end{array}$ & $\begin{array}{l}9,0 \\
7,5 \\
\end{array}$ & $\begin{array}{l}4,0 \\
4,0 \\
\end{array}$ & 0,123 \\
\hline $\begin{array}{l}\text { Semestre em curso } \\
\text { Antes do sétimo } \\
\text { Sétimo e oitavo } \\
\text { Nono e décimo }\end{array}$ & $\begin{array}{l}33 \\
27 \\
28 \\
\end{array}$ & $\begin{array}{l}6,90 \\
9,22 \\
9,42 \\
\end{array}$ & $\begin{array}{l} \pm 2,65 \\
\pm 2,04 \\
\pm 1,79\end{array}$ & $\begin{array}{r}6,0 \\
10,0 \\
9,5\end{array}$ & $\begin{array}{l}3,0 \\
4,0 \\
2,5\end{array}$ & $<0,001$ \\
\hline $\begin{array}{l}\text { Conteúdo sobre CSI* } \\
\text { Sim } \\
\text { Não }\end{array}$ & $\begin{array}{l}33 \\
55 \\
\end{array}$ & $\begin{array}{l}9,15 \\
7,98 \\
\end{array}$ & $\begin{array}{l} \pm 2,03 \\
\pm 2,65 \\
\end{array}$ & $\begin{array}{r}10,0 \\
8,0 \\
\end{array}$ & $\begin{array}{l}3,0 \\
4,0 \\
\end{array}$ & 0,032 \\
\hline $\begin{array}{l}\text { Conteúdo sobre } \text { ISTI }^{* *} \\
\text { Sim } \\
\text { Não }\end{array}$ & $\begin{array}{l}27 \\
61\end{array}$ & $\begin{array}{l}9,37 \\
8,00\end{array}$ & $\begin{array}{l} \pm 1,94 \\
\pm 2,60\end{array}$ & $\begin{array}{r}10,0 \\
8,0\end{array}$ & $\begin{array}{l}3,0 \\
4,0 \\
\end{array}$ & 0,016 \\
\hline $\begin{array}{l}\text { Atividades complementares como } \\
\text { palestra/workshop sobre CSI } \\
\text { Sim } \\
\text { Não }\end{array}$ & $\begin{array}{r}9 \\
79\end{array}$ & $\begin{array}{r}10,66 \\
8,16\end{array}$ & $\begin{array}{l} \pm 1,65 \\
\pm 2,45\end{array}$ & $\begin{array}{r}10,0 \\
8,0\end{array}$ & $\begin{array}{l}2,0 \\
4,0\end{array}$ & 0,003 \\
\hline
\end{tabular}


Research, Society and Development, v. 11, n. 3, e13311326110, 2022

(CC BY 4.0) | ISSN 2525-3409 | DOI: http://dx.doi.org/10.33448/rsd-v11i3.26110

\begin{tabular}{|c|c|c|c|c|c|c|}
\hline $\begin{array}{l}\text { Atividades complementares como } \\
\text { palestra/workshop sobre ISTI } \\
\text { Sim } \\
\text { Não }\end{array}$ & $\begin{array}{l}10 \\
78\end{array}$ & $\begin{array}{r}10,90 \\
8,10\end{array}$ & $\begin{array}{l} \pm 1,44 \\
\pm 2,42\end{array}$ & $\begin{array}{r}11,0 \\
8,0\end{array}$ & $\begin{array}{l}2,0 \\
4,0\end{array}$ & $<0,001$ \\
\hline $\begin{array}{l}\text { Pesquisa com pessoas idosas } \\
\text { Sim } \\
\text { Não }\end{array}$ & $\begin{array}{l}13 \\
75 \\
\end{array}$ & $\begin{array}{l}8,84 \\
8,34 \\
\end{array}$ & $\begin{array}{l} \pm 1,81 \\
\pm 2,59 \\
\end{array}$ & $\begin{array}{l}9,0 \\
9,0 \\
\end{array}$ & $\begin{array}{l}3,0 \\
4,0 \\
\end{array}$ & 0,508 \\
\hline $\begin{array}{l}\text { Extensão com pessoas idosas } \\
\text { Sim } \\
\text { Não }\end{array}$ & $\begin{array}{l}23 \\
65 \\
\end{array}$ & $\begin{array}{l}8,78 \\
8,29 \\
\end{array}$ & $\begin{array}{l} \pm 2,52 \\
\pm 2,49\end{array}$ & $\begin{array}{r}10,0 \\
9,0 \\
\end{array}$ & $\begin{array}{l}4,0 \\
4,0 \\
\end{array}$ & 0,421 \\
\hline $\begin{array}{llll}\text { Prática } & \text { assistencial com } & \text { pessoas } \\
\text { idosas } & & & \\
\text { Sim } & & & \\
\text { Não } & & & \\
\end{array}$ & $\begin{array}{r}80 \\
8\end{array}$ & $\begin{array}{l}8,58 \\
6,75\end{array}$ & $\begin{array}{l} \pm 2,49 \\
\pm 1,83\end{array}$ & $\begin{array}{l}9,0 \\
6,5\end{array}$ & $\begin{array}{l}3,5 \\
3,0\end{array}$ & 0,046 \\
\hline
\end{tabular}

*CSI: Comportamento sexual de pessoas idosas / **ISTI: Infecções sexualmente transmissíveis em pessoas idosas. Fonte: Autores (2020).

Os resultados do modelo final mostram a associação estatisticamente significativa entre o conhecimento e as seguintes variáveis: semestre em curso, palestra sobre IST, faixa etária e contato com idosos, ajustado por sexo. A maior média do escore de conhecimento sobre comportamento sexual e IST em pessoas idosas foi de acadêmicos de enfermagem que cursam do sétimo ao décimo semestre, que já participaram de atividades complementares (palestras/workshop) sobre IST de pessoas idosas, possuem idade acima de 27 anos e têm contato próximo com pessoas de 60 anos ou mais (Tabela 2).

Tabela 2. Modelo de regressão linear múltiplo: variáveis associadas ao conhecimento de acadêmicos de enfermagem sobre comportamento sexual e IST em pessoas idosas (n=88). Tangará da Serra, MT, 2020.

\begin{tabular}{|c|c|c|c|}
\hline Variáveis & $\begin{array}{c}\text { Coeficiente } \\
\text { regressão }\end{array}$ & IC $95 \%$ & $\mathbf{p}$ \\
\hline $\begin{array}{l}\text { Semestre em curso } \\
\text { Antes do sétimo } \\
\text { Sétimo e oitavo } \\
\text { Nono e décimo }\end{array}$ & $\begin{array}{c}1,0 \\
2,22 \\
1.67 \\
\end{array}$ & $\begin{array}{c}- \\
1.08-3.37 \\
0.52-2,82\end{array}$ & $\begin{array}{r}<0,001 \\
0,005\end{array}$ \\
\hline $\begin{array}{l}\text { Atividades complementares } \\
\text { palestra/workshop } \text { sobre ISTI } \\
\text { Sim } \\
\text { Não }\end{array}$ & $\begin{array}{c}2,43 \\
1,0 \\
\end{array}$ & $\begin{array}{c}1,01-3,84 \\
-\end{array}$ & 0,001 \\
\hline $\begin{array}{l}\text { Faixa etária } \\
22 \text { anos } \\
23 \text { a } 26 \text { anos } \\
27 \text { a } 39 \text { anos }\end{array}$ & $\begin{array}{c}1,0 \\
1,02 \\
1,45\end{array}$ & $\begin{array}{c}- \\
-1,08-1,04 \\
0,18-2,70 \\
\end{array}$ & $\begin{array}{l}0,970 \\
0,025 \\
\end{array}$ \\
\hline $\begin{array}{l}\text { Contato com pessoas idosas } \\
\text { Sim } \\
\text { Não }\end{array}$ & $\begin{array}{c}1,06 \\
1,0 \\
\end{array}$ & $\begin{array}{c}0,48-2,07 \\
- \\
\end{array}$ & 0,040 \\
\hline $\begin{array}{l}\text { Sexo* } \\
\text { Feminino } \\
\text { Masculino }\end{array}$ & $\begin{array}{c}-0,59 \\
1,0 \\
\end{array}$ & $\begin{array}{c}-2,08-0,88 \\
- \\
\end{array}$ & 0,424 \\
\hline
\end{tabular}

*Variável de ajuste. Fonte: Autores (2020).

$\mathrm{Na}$ análise bivariada entre atitude e variáveis sociodemográficas e de formação, verificou-se que as variáveis atividade complementar sobre ISTI e CSI apresentaram associação significativa. Assim, o escore de atitude foi maior entre os alunos que tiveram formação complementar (Tabela 3). 
Tabela 3. Média, mediana, desvio-padrão do escore de atitude e medidas de associação entre variáveis sociodemográficas e de formação (n=88). Tangará da Serra, MT, 2020.

\begin{tabular}{|c|c|c|c|c|c|c|}
\hline Variáveis & $\mathbf{n}$ & Média & $\begin{array}{l}\text { Desvio- } \\
\text { Padrão }\end{array}$ & Mediana & $\begin{array}{c}\text { Distância } \\
\text { interquartil }\end{array}$ & $\mathbf{p}$ \\
\hline $\begin{array}{l}\text { Sexo } \\
\text { Feminino } \\
\text { Masculino } \\
\end{array}$ & $\begin{array}{r}79 \\
9 \\
\end{array}$ & $\begin{array}{l}107,2 \\
103,8 \\
\end{array}$ & $\begin{array}{l} \pm 13,3 \\
\pm 13,2 \\
\end{array}$ & $\begin{array}{l}110 \\
102 \\
\end{array}$ & $\begin{array}{r}18,0 \\
8,0 \\
\end{array}$ & 0,479 \\
\hline $\begin{array}{l}\text { Faixa etária } \\
20 \text { a } 22 \text { anos } \\
23 \text { a } 26 \text { anos } \\
27 \text { a } 39 \text { anos } \\
\end{array}$ & $\begin{array}{l}36 \\
34 \\
18 \\
\end{array}$ & $\begin{array}{l}107,3 \\
105,1 \\
109,3 \\
\end{array}$ & $\begin{array}{l} \pm 13,3 \\
\pm 13,9 \\
\pm 12,0 \\
\end{array}$ & $\begin{array}{l}109 \\
106 \\
111 \\
\end{array}$ & $\begin{array}{l}18,5 \\
14,0 \\
19,0 \\
\end{array}$ & 0,553 \\
\hline $\begin{array}{l}\text { Estado civil } \\
\text { Sem companheiro } \\
\text { Com companheiro } \\
\end{array}$ & $\begin{array}{l}59 \\
29 \\
\end{array}$ & $\begin{array}{l}107,8 \\
104,9 \\
\end{array}$ & $\begin{array}{l} \pm 13,3 \\
\pm 13,2 \\
\end{array}$ & $\begin{array}{l}111 \\
107 \\
\end{array}$ & $\begin{array}{l}17,0 \\
14,0 \\
\end{array}$ & 0,347 \\
\hline $\begin{array}{l}\text { Religião } \\
\text { Católico } \\
\text { Protestante } \\
\text { Outra } \\
\text { Sem religião } \\
\end{array}$ & $\begin{array}{r}34 \\
34 \\
5 \\
15 \\
\end{array}$ & $\begin{array}{l}108,4 \\
103,5 \\
111,0 \\
109,5 \\
\end{array}$ & $\begin{array}{l} \pm 12,1 \\
\pm 14,9 \\
\pm 9,8 \\
\pm 12,2\end{array}$ & $\begin{array}{l}113 \\
107 \\
112 \\
111 \\
\end{array}$ & $\begin{array}{l}16,0 \\
16,0 \\
12,0 \\
21,0\end{array}$ & 0,303 \\
\hline $\begin{array}{l}\text { Contato próximo com pessoas } \\
\text { idosas } \\
\text { Sim } \\
\text { Não }\end{array}$ & $\begin{array}{l}64 \\
24 \\
\end{array}$ & $\begin{array}{l}105,5 \\
110,5 \\
\end{array}$ & $\begin{array}{l} \pm 13,3 \\
\pm 12,9 \\
\end{array}$ & $\begin{array}{l}107 \\
114 \\
\end{array}$ & $\begin{array}{l}16,0 \\
20,0 \\
\end{array}$ & 0,117 \\
\hline $\begin{array}{l}\text { Semestre em curso } \\
\text { Antes do sétimo } \\
\text { Sétimo e oitavo } \\
\text { Nono e décimo } \\
\end{array}$ & $\begin{array}{l}33 \\
27 \\
28 \\
\end{array}$ & $\begin{array}{l}105,2 \\
105,7 \\
109,9 \\
\end{array}$ & $\begin{array}{l} \pm 15,5 \\
\pm 11,3 \\
\pm 12,1 \\
\end{array}$ & $\begin{array}{l}107 \\
103 \\
112 \\
\end{array}$ & $\begin{array}{l}22,0 \\
14,0 \\
13,5 \\
\end{array}$ & 0,328 \\
\hline $\begin{array}{l}\text { Conteúdo sobre } \text { CSI }^{*} \\
\text { Sim } \\
\text { Não }\end{array}$ & $\begin{array}{l}33 \\
55 \\
\end{array}$ & $\begin{array}{l}106,6 \\
107,0 \\
\end{array}$ & $\begin{array}{l} \pm 15,2 \\
\pm 12,1 \\
\end{array}$ & $\begin{array}{l}111 \\
107 \\
\end{array}$ & $\begin{array}{l}18,0 \\
17,0 \\
\end{array}$ & 0,884 \\
\hline $\begin{array}{l}\text { Conteúdo sobre ISTI** } \\
\text { Sim } \\
\text { Não }\end{array}$ & $\begin{array}{l}27 \\
61 \\
\end{array}$ & $\begin{array}{l}108,6 \\
106,1\end{array}$ & $\begin{array}{l} \pm 13,1 \\
\pm 13,4\end{array}$ & $\begin{array}{l}112 \\
107\end{array}$ & $\begin{array}{l}19,0 \\
17,0\end{array}$ & 0,412 \\
\hline $\begin{array}{l}\text { Atividades complementares: } \\
\text { palestra/workshop sobre CSI* } \\
\text { Sim } \\
\text { Não }\end{array}$ & $\begin{array}{r}9 \\
79 \\
\end{array}$ & $\begin{array}{l}116,7 \\
105,7 \\
\end{array}$ & $\begin{array}{l} \pm 6,8 \\
\pm 13,4\end{array}$ & $\begin{array}{l}120 \\
107 \\
\end{array}$ & $\begin{array}{r}8,0 \\
16,0 \\
\end{array}$ & 0,017 \\
\hline $\begin{array}{l}\text { Atividades complementares: } \\
\text { palestra/workshop sobre ISTI** } \\
\text { Sim } \\
\text { Não }\end{array}$ & $\begin{array}{l}10 \\
78 \\
\end{array}$ & $\begin{array}{l}115,6 \\
105,7 \\
\end{array}$ & $\begin{array}{l} \pm 7,3 \\
\pm 13,5 \\
\end{array}$ & $\begin{array}{l}118 \\
107 \\
\end{array}$ & $\begin{array}{l}11,0 \\
17,0 \\
\end{array}$ & 0,026 \\
\hline $\begin{array}{l}\text { Pesquisa com pessoas idosas } \\
\text { Sim } \\
\text { Não }\end{array}$ & $\begin{array}{l}13 \\
75\end{array}$ & $\begin{array}{l}109,6 \\
106,4\end{array}$ & $\begin{array}{l} \pm 9,1 \\
\pm 13,8\end{array}$ & $\begin{array}{l}108 \\
109\end{array}$ & $\begin{array}{l}15,0 \\
17,0\end{array}$ & 0,423 \\
\hline $\begin{array}{l}\text { Extensão com pessoas idosas } \\
\text { Sim } \\
\text { Não }\end{array}$ & $\begin{array}{l}23 \\
65 \\
\end{array}$ & $\begin{array}{l}106,0 \\
107,2 \\
\end{array}$ & $\begin{array}{l} \pm 15,0 \\
\pm 12,7 \\
\end{array}$ & $\begin{array}{l}112 \\
107 \\
\end{array}$ & $\begin{array}{l}15,0 \\
18,0 \\
\end{array}$ & 0,715 \\
\hline $\begin{array}{l}\text { Prática assistencial com pessoas } \\
\text { idosas } \\
\text { Sim } \\
\text { Não }\end{array}$ & $\begin{array}{r}80 \\
8\end{array}$ & $\begin{array}{l}106,7 \\
108,6\end{array}$ & $\begin{array}{l} \pm 13,5 \\
\pm 10,8\end{array}$ & $\begin{array}{l}108 \\
110\end{array}$ & $\begin{array}{l}18,0 \\
11,0\end{array}$ & 0,698 \\
\hline
\end{tabular}

*CSI: Comportamento sexual de pessoas idosas / **ISTI: Infecções sexualmente transmissíveis em pessoas idosas. Fonte: Autores (2020).

Ao ajustar o modelo múltiplo com as variáveis atividades complementares sobre CS e IST, sexo e contato próximo com pessoas idosas, apenas a variável atividade complementar sobre CSI permaneceu estatisticamente significativa. As variáveis atividade complementar sobre CS e IST apresentam colinearidade e, por isso, foram ajustados modelos separados. 
Acadêmicos de enfermagem que participaram de atividades complementares sobre CSI apresentaram atitudes mais positivas sobre o comportamento sexual e as IST em pessoas idosas (Tabela 4).

Tabela 4. Modelo de regressão linear múltiplo: variáveis associadas à atitude de acadêmicos de enfermagem sobre comportamento sexual e IST em pessoas idosas (n=88). Tangará da Serra, MT, 2020.

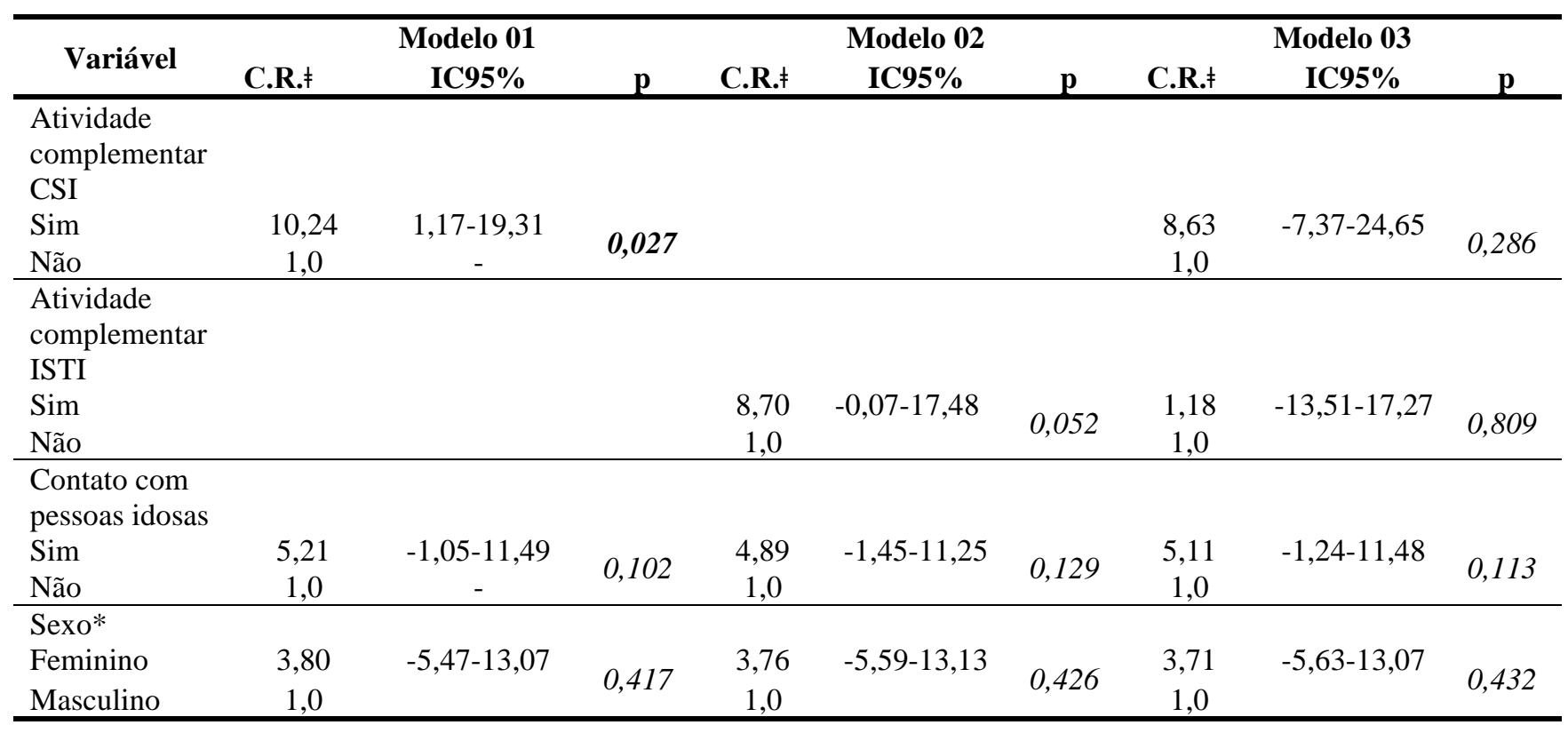

‡ Coeficiente de Regressão *Variável de ajuste. Fonte: Autores (2020).

\section{Discussão}

A relevância deste estudo consiste no aumento das evidências acerca do conhecimento e das atitudes de acadêmicos de enfermagem relacionado ao comportamento sexual de idosos e às IST. Seus resultados demonstram associação entre o conhecimento dos acadêmicos com variáveis sociodemográficas e de formação acadêmica na análise múltipla, enquanto a atitude associou-se somente com atividade complementar sobre CS.

Estudos observacionais realizados com acadêmicos da área da saúde também encontraram que os mais velhos, quando comparados aos mais jovens, tinham maior conhecimento sobre sexualidade de pessoas idosas (Gewirtz-Meydan et al., 2018; Folasayo et al., 2017). De fato, alunos mais velhos, provavelmente, são os que tiveram mais oportunidades de vivenciar ou participar de momentos que abordassem o tema e, portanto, tiveram maior oportunidade de adquirir novos conhecimentos, inclusive sobre o comportamento sexual de pessoas idosas.

Alguns estudos também evidenciaram a associação encontrada neste estudo entre conhecimento e contato com pessoas idosas (Chu et al., 2020; Gewirtz-Meydan et al., 2018). Estudos sobre a percepção dos jovens em relação às pessoas idosas e à velhice mostram que eles têm ideias equivocadas e com estereótipos culturais (Caldas et al., 2010; Neri, 2020). Entretanto, o indivíduo adquire conhecimentos e habilidades durante a troca de experiências no cotidiano, nas relações com as pessoas e com o meio (Alcântara et al., 2016). Ademais, o convívio com pessoas idosas influencia positivamente na percepção acerca do envelhecimento (Gvozd et al., 2012). Nesse sentido, pode ser que o contato dos acadêmicos com pessoas mais velhas tenha modificado essa percepção estereotipada.

A associação entre o conhecimento e as variáveis semestre em curso e participar de atividades complementares, provavelmente, pode ter ocorrido porque quanto maior o tempo na universidade, mais oportunidades existem para os alunos adquirirem conhecimentos. Corroborando os resultados deste estudo, algumas pesquisas evidenciaram que acadêmicos de 
graduação da área da saúde que cursaram maior tempo da faculdade tiveram maior conhecimento sobre a sexualidade na velhice, quando comparados aos alunos que cursam os semestres iniciais (Wilschut et al., 2021; Folasayo et al., 2017).

Esse achado não surpreende, pois geralmente os acadêmicos dos últimos anos da graduação já cursaram um maior número de disciplinas e podem ter tido acesso a conteúdo teóricos e práticos relacionados à população idosa. Nas Diretrizes Curriculares Nacionais (DCN) dos cursos de graduação em Enfermagem, atividades complementares (AC) devem, obrigatoriamente, compor sua estrutura curricular (Brasil, 2001). Essas atividades possibilitam ampliação de conhecimento profissional e permitem o desenvolvimento de competências técnicas e habilidades dos acadêmicos em áreas específicas como a gerontologia (Ferreira et al., 2016; Warmling et al., 2012). Esse é o caso dos participantes desta pesquisa, que cursaram a disciplina de saúde do idoso no sétimo semestre do curso e participaram de AC.

Contrariamente ao que era esperado, não houve associação entre as atitudes de acadêmicos de enfermagem sobre o comportamento sexual e as IST em pessoas idosas e as variáveis sociodemográficas. Outro estudo também não encontrou associação entre atitudes de acadêmicos sobre esse tema com as variáveis sexo e idade (Ouzouni et al., 2012).

A ausência de associação entre essas variáveis não tem explicação clara na literatura. Possivelmente, a crença de que pessoas idosas não têm vida sexual ativa tenha influência tanto em acadêmicos do sexo feminino quanto masculino, bem como em acadêmicos mais jovens e mais velhos, independente de terem tido contato com pessoas idosas. Estudo mostrou que acadêmicos de enfermagem com crenças negativas sobre a saúde sexual têm atitudes negativas (Öz et al., 2021). Mitos afetam as atitudes sobre sexualidade (Akalin et al., 2020).

Quanto à formação acadêmica, diferente de outra pesquisa ${ }^{21}$, os acadêmicos deste estudo que tiveram $\mathrm{AC}$ ou treinamento sobre o comportamento sexual de pessoas idosas apresentaram atitudes mais positivas em relação ao tema. Pode ser que o fato desses alunos terem acesso ao conteúdo específico sobre o comportamento sexual de pessoas idosas durante essas atividades tenha contribuído para influenciar suas atitudes. Estudos apontam que acadêmicos da área da saúde com melhor conhecimento sobre saúde sexual de pessoas idosas têm atitudes mais positivas (Gewirtz-Meydan et al., 2018; Sung et al., 2015). Ademais, o componente cognitivo das atitudes envolve o conhecimento que o indivíduo tem em relação ao objeto atitudinal (Folasayo et al., 2017).

Investigar essas temáticas no contexto da formação de acadêmicos de enfermagem é importante, uma vez que tabus e estereótipos sobre o assunto são evidentes na sociedade, nas universidades e entre os profissionais de saúde. Além disso, é preciso garantir que esses conteúdos sejam contemplados em sua complexidade nos currículos dos cursos, dando essas especificidades, a fim de que esses estereótipos sejam minimizados.

Assim, deve-se refletir sobre os processos formativos de enfermeiros e desenvolver estratégias que favoreçam discussões e reflexões para a recondução de valores e conceitos estabelecidos, capazes de impactar no futuro profissional de acadêmicos. Recomenda-se que nos currículos de enfermagem sejam incluídos tópicos específicos do processo de envelhecimento e envelhecer, contribuindo para o desenvolvimento de atitudes e comportamentos favoráveis sobre a sexualidade e a saúde sexual, incluindo a prevenção das IST.

Uma limitação deste estudo é que a amostra foi de acadêmicos de enfermagem de apenas um curso e vinculados a uma universidade pública. Portanto, seus resultados não podem ser generalizados. Ademais, outra limitação é aplicação de questionário online como técnica para a coleta de dados, pois pode permitir maior número de recusas ou perdas de participantes e reduzir a amostra do estudo. Pesquisas futuras devem ocorrer com maior número de acadêmicos de universidades públicas e privadas, englobando outros desenhos metodológicos. 


\section{Conclusão}

Os resultados deste estudo demonstram associação do conhecimento de acadêmicos de enfermagem sobre o comportamento sexual e as IST em pessoas idosas com variáveis sociodemográficas e de formação acadêmica. Acadêmicos com idade acima de 27 anos, que têm contato próximo com pessoas idosas, cursam a partir do terceiro ano da graduação e tiveram AC sobre saúde sexual de pessoas idosas expressam maior conhecimento. No que se refere às atitudes, os resultados revelam que acadêmicos de enfermagem que tiveram AC sobre saúde sexual de pessoas idosas possuem atitudes mais positivas em relação ao comportamento sexual e às IST nessa população.

\section{Referências}

Agochukwu-Mmonu, N., Malani, P.N., Wittmann, D., Kirch M., Kullgren J., Singer D., \& Solway E. (2021). Interest in Sex and Conversations About Sexual Health with Health Care Providers Among Older US Adults. Clinical Gerontologist, 44(3), 299-306.

Akalin, A. \& Ozkan, B. (2020). Sexual myths and attitudes regarding sexuality of nursing students: A mixed method study. Perspectives in psychiatric care, 57(3), 1497-1504.

Alcântara, A. D. O. O., Camarano, A. A. O., \& Giacomin, K. C. O. (2016). Política Nacional do Idoso: velhas e novas questões.

Altmann, T. K. (2008). Attitude: a concept analysis. In Nursing forum (Vol. 43, No. 3, pp. 144-150). Malden, USA: Blackwell Publishing Inc.

Andrade, J., Ayres, J. A., Alencar, R. A., Duarte, M. T. C., \& Parada, C. M. G. D. L. (2017). Vulnerabilidade de idosos a infecções sexualmente transmissíveis. Acta Paulista de Enfermagem, 30, 8-15.

Brasil. RESOLUÇÃO CNE/CES Nº 5, DE 7 DE NOVEMBRO DE 2001.

Caldas, C. P., \& Thomaz, A. F. (2010). A Velhice no Olhar do Outro: Uma perspectiva do jovem sobre o que é ser velho. Revista Kairós: Gerontologia, 13(2).

Cappiello, J., Coplon, L., \& Carpenter, H. (2017). Systematic review of sexual and reproductive health care content in nursing curricula. Journal of Obstetric, Gynecologic \& Neonatal Nursing, 46(5), e157-e167.

Linley, L., Atkins, C. Y., Li, J., Yu, C., Johnson, A. S., \& Friend, M. (2018). Diagnoses of HIV infection among adults aged 50 years and older in the United States and dependent areas 2011-2016.

Chu, C. W., \& Wang, J. J. (2012). Nursing students' knowledge of and attitudes toward sexuality in the elderly. Journal of Nursing and Healthcare Research, $8(4), 306-315$.

da Costa, D. C. A., da Silva Uchôa, Y., da Silva Junior, I. A. P., de Matos Freitas, W. M. T., \& Soares, S. C. (2017). Sexualidade no idoso: percepção de profissionais da geriatria e gerontologia. Universitas: Ciências da Saúde, 15(2), 75-80.

Cunha, L. M., Mota, W. S., Gomes, S. C., Bezerra, Í. M. P., Machado, M. D. F. A. S., \& Quirino, G. D. S. (2015). Vovó e vovô também amam: sexualidade na terceira idade. Revista Mineira de Enfermagem, 19(4), 894-906.

Dos Reis, R. P., Oliveira, J. K. C., Vanderlei, M. G., Barbosa, D. F. R., Dos Santos, J. M., Gomes, M. P., ... \& Bezerra, D. G. (2020). A atuação do enfermeiro frente à sexualidade na terceira idade: uma revisão integrativa. Revista Eletrônica Acervo Saúde, (55), e3740-e3740.

European Centre for Disease Prevention And Control [ECDPC]. (2020). Syphilis. Annual epidemiological report for 2018. Stockholm.

Evangelista, A. D. R., Moreira, A. C. A., Freitas, C. A. S. L., Val, D. R. D., Diniz, J. L., \& Azevedo, S. G. V. (2019). Sexualidade de idosos: conhecimento/atitude de enfermeiros da Estratégia Saúde da Família. Revista da Escola de Enfermagem da USP, 53.

Ferreira, I. G., Carreira, L. B., Botelho, N. M., \& de Souza, L. E. A. (2016). Atividades extracurriculares e formação médica: diversidade e flexibilidade curricular. IJHE-Interdisciplinary Journal of Health Education, 1(2).

Folasayo, A. T., Oluwasegun, A. J., Samsudin, S., Saudi, S. N. S., Osman, M., \& Hamat, R. A. (2017). Assessing the knowledge level, attitudes, risky behaviors and preventive practices on sexually transmitted diseases among university students as future healthcare providers in the central zone of Malaysia: a cross-sectional study. International journal of environmental research and public health, 14(2), 159.

Gaspar, V. S., da Silva Brito, J. H., \& do Nascimento, D. E. M. (2020). Saúde sexual na terceira idade: o desafio de compreender as vivências. Brazilian Journal of Health Review, 3(5), 13109-13125.

Gewirtz-Meydan, A., Even-Zohar, A., \& Werner, S. (2018). Examining the attitudes and knowledge of social work and nursing students on later-life sexuality. Canadian Journal on Aging/La Revue canadienne du vieillissement, 37(4), 377-389.

Gvozd, R., \& Dellaroza, M. S. G. (2012). Velhice e a relação com idosos: o olhar de adolescentes do ensino fundamental. Revista Brasileira de Geriatria e Gerontologia, 15, 295-304.

Madruga, M. D. D., Vieira, K. F. L., \& de Almeida, S. A. (2018). Fatores de vulnerabilidade dos idosos ao hiv/aids: uma revisão integrativa. Revista de Pesquisa Cuidado é Fundamental Online, 10, 12-18. 
Mahmud, I. C., Cunha, L. D. A. D., Behar, P. R. P., \& Terra, N. L. (2021). O desafio do HIV em idosos: uma análise qualitativa da atuação de médicos da atenção primária à saúde em Porto Alegre/RS. Rev. Pesqui.(Univ. Fed. Estado Rio J., Online), 384-390.

Ministério da Saúde. (2020). Secretaria de Vigilância em Saúde. Boletim Epidemiológico de HIV e Aids.

Ministério da Saúde. (2021). Secretaria de Vigilância em Saúde. Boletim Epidemiológico de Sífilis.

Moreira, W. C., Carvalho, A. R. B. D., Lago, E. C., Amorim, F. C. M., Alencar, D. D. C., \& Almeida, C. A. P. L. (2018). Formação de estudantes de Enfermagem para atenção integral ao idoso. Revista Brasileira de Geriatria e Gerontologia, 21, 186-193.

Moura, D. S., Pessôa, R. M. C., \& Almeida, M. M. (2017). Sexuality in the elderly: a discussion about the measures of prevention of HIV/aids. ReOn Facema, 3(1), 407-415.

Ouzouni, C., \& Nakakis, K. (2012). HIV/AIDS knowledge, attitudes and behaviours of student nurses. Health Science Journal, 6(1), 129.

Öz, H. G., Yangın, H. B., \& Sözer, G. A. (2021). Attitudes and beliefs of nursing students toward sexual healthcare: A descriptive study. Perspectives in psychiatric care.

Neri, A. L. (2020). Idosos no Brasil II: vivências, desafios e expectativas na terceira idade. São Paulo: Fundação Perseu Abramo.

Sung, S. C., Huang, H. C., \& Lin, M. H. (2015). Relationship between the knowledge, attitude, and self-efficacy on sexual health care for nursing students. Journal of Professional Nursing, 31(3), 254-261.

Venturini, L., Beuter, M., Leite, M. T., Bruinsma, J. L., \& Backes, C. (2018). Atuação da equipe de enfermagem frente à sexualidade de idosas institucionalizadas. Revista da Escola de Enfermagem da USP, 52.

Warmling, A. M. F., de Mello, A. L. S. F., Naspolini, D. S., de Luca Canto, G., \& de Souza, E. R. (2012). Contribuições das atividades complementares na formação. Revista da ABENO, 12(2), 190-197.

Wilschut, V. F., Pianosi, B., van Os-Medendorp, H., Elzevier, H. W., Jukema, J. S., \& den Ouden, M. E. (2021). Knowledge and attitude of nursing students regarding older adults' sexuality: A cross-sectional study. Nurse Education Today, 96, 104643.

Yang, S., Yan, E., \& Bai, X. (2018). An integrated model to understand sexuality in older adults in urban China: The mediating role of sexual interest. Archives of gerontology and geriatrics, 78, 51 